\title{
Higher-Order Sliding Mode Control for Lateral Dynamics of Autonomous Vehicles, with Experimental Validation
}

\author{
Gilles Tagne, Reine Talj and Ali Charara
}

\begin{abstract}
This paper presents design and experimental validation of a vehicle lateral controller for autonomous vehicle based on a higher-order sliding mode control. We used the super-twisting algorithm to minimize the lateral displacement of the autonomous vehicle with respect to a given reference trajectory. The control input is the steering angle and the output is the lateral displacement error. The particularity of such a strategy is to take advantage of the robustness of the sliding mode controller against nonlinearities and parametric uncertainties in the model, while reducing chattering, the main drawback of first order sliding mode. To validate the control strategy, the closed-loop system simulated on MatlabSimulink has been compared to the experimental data acquired on our vehicle DYNA, a Peugeot 308, according to several driving scenarios. The validation shows robustness and good performance of the proposed control approach.
\end{abstract}

\section{INTRODUCTION}

Technological advances in recent years have favored the emergence of intelligent vehicles with the capacity to anticipate and compensate a failure (of driver, vehicle or infrastructure) or even to ensure an autonomous driving.

The "DARPA Grand Challenge" $(2004,2005)$ and the "DARPA Urban Challenge" (2007) [1], organized by the Defense Advanced Research Projects Agency (DARPA) of the U.S. has stimulated research for the development of autonomous vehicles. This is an area of growing research. One of the major challenges today is to ensure autonomous driving at high speed.

An autonomous driving can be divided in three steps:

- The perception of the environment. It consists on detecting road, obstacles and other vehicles. A vision system composed of sensors like cameras, lasers, radars and GPS is usually used to achieve this goal. It provides a dynamic map of the near environment of the autonomous vehicle.

- The trajectory generation. It consists in generating and choosing one trajectory (reference path) in the navigable space.

- The vehicle control. It consists to handle vehicle using actuators like brake, accelerator and steering wheel to follow the reference path. This step can be divided into two tasks: longitudinal control and lateral control.

This paper focus on the third main step, that treat the vehicle control. And more precisely, the lateral control of the

Gilles Tagne, Reine Talj and Ali Charara are working at Heudiasyc Laboratory, UMR CNRS 7253, Université de Technologie de Compiègne, BP 20529, 60205 Compiègne, France.

gilles.tagne@hds.utc.fr, reine.talj@hds.utc.fr, ali.chararaehds.utc.fr intelligent vehicle. Lateral control of an autonomous vehicle deal with automatically steering the vehicle to follow the reference path. It has been studied since the 1950s. Given the high nonlinearities of the vehicle system on one hand, and the uncertainties and disturbances of such a system on the other hand, a very important issue to be considered in the control design is the robustness. The controller should be able to reject the disturbances caused by wind, coefficient of friction of the road and many other reasons, and able to deal with parameter uncertainties and variations encountered in automotive applications. For example, in [2], a recent presentation of Junior; Stanford's autonomous research vehicle (the second at the DARPA Urban Challenge) is made for the purpose of ensuring robust autonomous driving.

For over 40 years, considerable research have been conducted to provide lateral guidance of autonomous vehicles. Several control strategies have been developed in the literature: In [3], proportional controller is used. In [4], PI controller is used. In [5], nested PID controller is proposed. In [6], controller based on state feedback control is developed. In [7], the $H_{\infty}$ control is used. In [8], control by Lyapunov stability theory is developed. In [9], a controller based on adaptive control is presented. In [10], [11], controllers based on linear quadratic optimal predictive control are developed.

In [12], techniques from artificial intelligence and fuzzy logic are particularly used. These approaches use human steering skills to improve the automatic driving performance.

In [13], a comparison of a proportional, adaptive, $H_{\infty}$ and fuzzy controllers is presented. This comparison is made using several criteria to evaluate the robustness: variations of curvature, speed variations, changes of the friction coefficient of the road and disturbances due to wind. From this comparison, it appears that the proportional controller is the one that has the biggest errors. $H_{\infty}$ and fuzzy controllers have equivalent performances. The adaptive controller has the best response.

Model predictive control (MPC) appears to be well suited to the trajectory following [2], [14]. It allows to consider the problem of trajectory tracking for nonlinear systems taking into account the constraints on the state variables and/or control inputs. In addition, this control technique is proved to be robust against system parameter variations. However, for autonomous driving at high speed, the computation time (non-linear optimization algorithms) becomes very large for real-time operation [10], [14].

Different comparisons showed that the class of adaptive controllers represents a very promising technique for such uncertain and nonlinear application. 
The sliding mode control is one of the most promising control techniques for trajectory tracking. In [15], [16], [17], the first-order sliding mode control is applied to the lateral control. This control strategy is well suited for driving applications. It provides constant and small displacement errors when the speed increases. Also, it allows to obtain experimental results comparable or better than the linear controllers with gain self-adjusting [16]. In addition, it is particularly suited to compensate for uncertainties (variations of model parameters) and disturbances encountered in automotive applications. This method also has the advantage of producing simpler control laws lower complexity compared to other approaches of robust control [18]. However, their main drawback is the chattering. One of the solutions to reduce the chattering is the using of higher-order sliding mode.

We propose in this paper a controller based on higherorder sliding mode, the algorithm of super-twisting to ensure lateral control at high speed of an autonomous vehicle. The problem considers that the vehicle is equipped with all the necessary sensors to measure or estimate lateral acceleration, lateral velocity, yaw rate and steering angle. To validate the proposed approach, tests were made with real data acquired by our vehicle DYNA, on the tracks and circuits of CERAM $^{1}$. The experimental results show the effectiveness of the proposed approach.

This paper is organized as follows. Sections II presents the dynamic models of the vehicle that we used. In Section III, we develop our control strategy. Section IV presents the results and the evaluation of robustness. Section $\mathrm{V}$ presents the conclusions, remarks and future work directions.

\section{DYNAMIC MODELS OF VEHICLE}

In this work, we use two vehicle models. The first one is the bicycle model used in Section III.B for the control design. The second is the 4-wheel vehicle model used to validate in simulation the proposed control in closed loop.

\section{A. Bicycle model}

To design the controller, a simple and widely used dynamic bicycle model [6] is considered. See Fig. 1. This

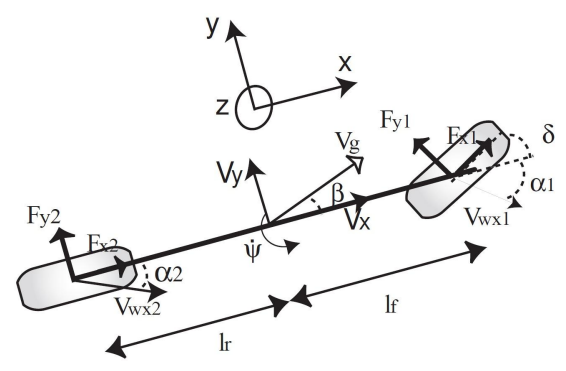

Fig. 1. Bicycle model

model is used to represent the lateral vehicle behavior (lateral acceleration, yaw rate, sideslip angle) and assumes that the

\footnotetext{
${ }^{1}$ CERAM -'Centre d'Essais et de Recherche Automobile de Mortefontaine" is an automobile testing and research center located in France.
}

vehicle is symmetrical, and sideslip angles on the same axle are equal. The roll and pitch dynamics are neglected and angles are assumed to be small (steering, sideslip, yaw).

With a linear tire force model we obtain a linear parameter varying model (LPV), the longitudinal velocity $V_{x}$ is considered as a varying parameter. This LPV model is composed of the lateral and yaw dynamics given by:

$$
\left\{\begin{array}{l}
\ddot{y}=-\frac{\left(C_{f}+C_{r}\right)}{m V_{x}} \dot{y}-\left(\frac{L_{f} C_{f}-L_{r} C_{r}}{m V_{x}}+V_{x}\right) \dot{\psi}+\frac{C_{f}}{m} \delta \\
\ddot{\psi}=-\frac{L_{f} C_{f}-L_{r} C_{r}}{I_{z} V_{x}} \dot{y}-\frac{L_{f}^{2} C_{f}+L_{r}^{2} C_{r}}{I_{z} V_{x}} \dot{\psi}+\frac{L_{f} C_{f}}{I_{z}} \delta
\end{array}\right.
$$

where $y$ and $\psi$ represent respectively the lateral position and the yaw angle of the vehicle. Table I presents vehicle nomenclature and parameters.

TABLE I

Vehicle Nomenclature And Parameters (Bicycle Model)

\begin{tabular}{|c||c||c||c|}
\hline$V_{x}$ & Longitudinal velocity & - & {$[\mathrm{m} / \mathrm{s}]$} \\
\hline$\dot{y}$ & Lateral velocity & - & {$[\mathrm{m} / \mathrm{s}]$} \\
\hline$\dot{\psi}$ & Yaw rate & - & {$[\mathrm{rad} / \mathrm{s}]$} \\
\hline$\delta$ & steering wheel angle & - & {$[\mathrm{rad}]$} \\
\hline $\mathrm{m}$ & Mass & 1719 & {$[\mathrm{~kg}]$} \\
\hline$I_{z}$ & Yaw moment of inertia & 3300 & {$\left[\mathrm{kgm}^{2}\right]$} \\
\hline$L_{f}$ & Front axle-COG distance & 1.195 & {$[\mathrm{~m}]$} \\
\hline$L_{r}$ & Rear axle-COG distance & 1.513 & {$[\mathrm{~m}]$} \\
\hline$C_{f}$ & Cornering stiffness of the front tire & 170550 & {$[\mathrm{~N} / \mathrm{rad}]$} \\
\hline$C_{r}$ & Cornering stiffness of the rear tire & 137844 & {$[\mathrm{~N} / \mathrm{rad}]$} \\
\hline
\end{tabular}

\section{B. 4-wheel model}

To compare our simulation results with experimental data, we used a more representative model. Namely, a 4-wheel model to represent the vehicle dynamics, with Dugoff's tire model for logitudinal and lateral forces [19].

\section{CONTROL STRATEGY}

The sliding mode control (SMC) has been developed since 1950s and is recognized as one of the most promising techniques for robust control. The principle of SMC is to constrain the system trajectories to reach in finite time and remain on a sliding surface (see Fig. 2). However, its main

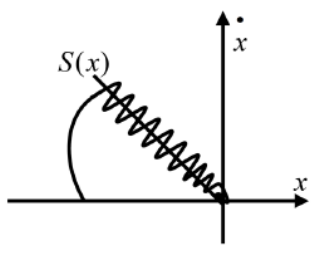

Fig. 2. SMC principle

drawback is the chattering. Three main approaches to the elimination and mitigation of chattering in the SMC have been proposed in the mid 1980s [20]:

- The use of smooth functions instead of the discontinuous function sign,

- The use of an approach based on observers,

- The use of higher order sliding mode. 


\section{A. Super-twisting algorithm}

The super-twisting algorithm is developed to control systems with a relative degree 1 , and to ensure robust stability while reducing chattering (the main drawback of the firstorder sliding mode). Consider a system of the form:

$$
\dot{x}=f(t, x)+g(t, x) u(t)
$$

where $u$ is the control input, $x \in \mathbb{R}^{n}$ the state vector, and, $f, g$ continuous functions. We define a sliding variable $s$ of relative degree 1 , whose derivative can be expressed as follows:

$$
\dot{s}(t, s)=\phi(t, s)+\varphi(t, s) u(t)
$$

The aim of the controller is to ensure converge to the sliding surface defined by $s=0$. Only the measurement of $s$ in real time is required.

It is assumed that there exist positive constants $s_{0}, b_{\min }, b_{\max }$, $C_{0}$ such that $\forall x \in \mathbb{R}^{n}$ and $|s(t, x)|<s_{0}$, the system satisfies the following conditions:

$$
\left\{\begin{array}{l}
|u(t)| \leq U_{\max } \\
0<b_{\min } \leq|\varphi(t, s)| \leq b_{\max } \\
|\phi(t, s)|<C_{0}
\end{array}\right.
$$

The sliding mode control algorithm based on super-twinting is given by:

$$
u(t)=u_{1}+u_{2}\left\{\begin{array}{l}
\left.\left.u_{1}=-\alpha|s|^{\tau} \operatorname{sign}(s), \quad \tau \in\right] 0,0.5\right] \\
\dot{u}_{2}=-\beta \operatorname{sign}(s)
\end{array}\right.
$$

with $\alpha$ and $\beta$ positive constants. The finite time convergence to the sliding surface is guaranteed by the following conditions [21]:

$$
\left\{\begin{array}{l}
\beta>\frac{C_{0}}{b_{\min }} \\
\alpha \geq \sqrt{\frac{4 C_{0}\left(b_{\max } \beta+C_{0}\right)}{b_{\min }^{2}\left(b_{\min } \beta-C_{0}\right)}}
\end{array}\right.
$$

For more details of the convergence and robustness of the algorithm, see [22], [23].

\section{B. Application to lateral control of autonomous vehicles}

The lateral error dynamics at the center of gravity of the vehicle, with respect to a reference trajectory, is given by:

$$
\ddot{e}=a_{y}-a_{y_{r e f}}
$$

where $a_{y}$ and $a_{y_{\text {ref }}}$ are the lateral acceleration of the vehicle, and the desired one on the reference trajectory respectively. Assuming that the desired lateral acceleration of the vehicle can be written as $a_{y_{\text {ref }}}=V_{x}^{2} / R$, where $R$ is the radius of curvature of the road and given that $a_{y}=\ddot{y}+V_{x} \dot{\psi}$, we have:

$$
\ddot{e}=\ddot{y}+V_{x} \dot{\psi}-\frac{V_{x}^{2}}{R}
$$

Replacing $\ddot{y}$ by its expression in equation (1), we obtain:

$$
\ddot{e}=-\frac{\left(C_{f}+C_{r}\right)}{m V_{x}} \dot{y}-\frac{L_{f} C_{f}-L_{r} C_{r}}{m V_{x}} \dot{\psi}-\frac{V_{x}^{2}}{R}+\frac{C_{f}}{m} \boldsymbol{\delta}
$$

The control input is the steering angle and the lateral displacement is the output. The objective of the control law is to cancel the lateral displacement error.
Choosing the sliding variable $s$ as follows:

$$
s=\dot{e}+\lambda e
$$

we obtain: $\dot{s}=\ddot{e}+\lambda \dot{e}$. Replacing $\ddot{e}$ by its expression (11), we obtain:

$$
\dot{s}=-\frac{C_{f}+C_{r}}{m V_{x}} \dot{y}-\frac{L_{f} C_{f}-L_{r} C_{r}}{m V_{x}} \dot{\psi}-\frac{V_{x}^{2}}{R}+\frac{C_{f}}{m} \boldsymbol{\delta}+\lambda \dot{e}
$$

The variable $s$ has a relative degree $r=1$. By identification with (3), we have $\dot{s}(t, s)=\phi(t, s)+\varphi(t, s) u(t)$, with:

$$
\left\{\begin{array}{l}
\phi(t, s)=-\frac{C_{f}+C_{r}}{m V_{x}} \dot{y}-\frac{L_{f} C_{f}-L_{r} C_{r}}{m V_{x}} \dot{\psi}-\frac{V_{x}^{2}}{R}+\lambda \dot{e} \\
\varphi(t, s)=\frac{C_{f}}{m}
\end{array}\right.
$$

Applying the super-twisting theorem, the control input can be defined as follows:

$$
\delta_{S T}=u_{1}+u_{2}\left\{\begin{array}{l}
u_{1}=-\alpha|s|^{1 / 2} \operatorname{sign}(s) \\
\dot{u}_{2}=-\beta \operatorname{sign}(s)
\end{array}\right.
$$

To avoid important peaks in transient phases, we add an equivalent command $\delta_{e q}$ obtained by solving the equation $\dot{s}=0$. This term has the role of a feedforward that approach the system to the sliding surface, and is given by:

$$
\delta_{e q}=-\frac{m}{C_{f}} \phi(t, s)
$$

Hence, the steering angle representing the control input of the system is defined as follows:

$$
\delta=\delta_{S T}+\delta_{e q}
$$

\section{EXPERIMENTAL VALIDATION}

The experimental data used here are acquired on the CERAM test circuits by our vehicle DYNA (Fig. 3). This vehicle is equipped with several sensors: an Inertial Measurement Unit (IMU) measuring accelerations $(\mathrm{x}, \mathrm{y}, \mathrm{z})$ and the yaw rate. The CORREVIT for measuring the sideslip angle and longitudinal velocity. Torque hubs for measuring tire-road efforts and vertical loads on each tire. Four laser sensors to measure the height of the chassis. GPS and a CCD camera. Data provided via the CAN bus of the vehicle are also used, as the steering angle, and the rotational speed of the wheels.

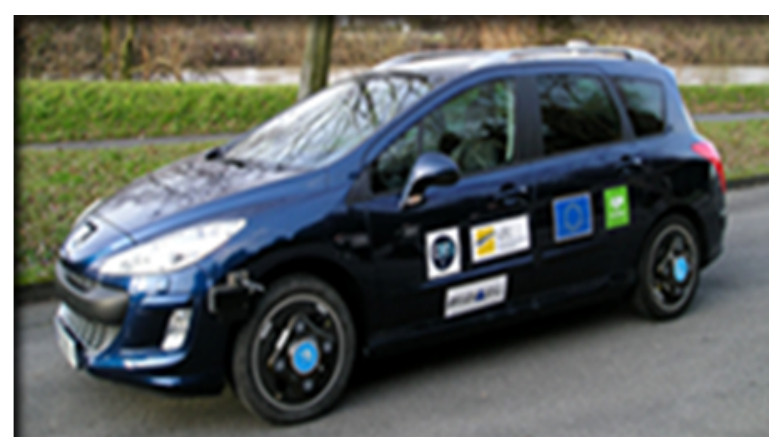

Fig. 3. Experimental vehicle (DYNA)

To validate our control law, we perform several tests of our vehicle DYNA. The collected data are reference data that will be compared to those obtained using simulations of the law 
of closed loop control. Simulations were carried out with our vehicle full model. For the control law, we used $\lambda=8, \alpha=$ $0.002, \beta=0.0001$ and the nominal vehicle parameters (see Table 1). The robustness of the controlled system is evaluated in terms of changes in parameters (longitudinal speed) and uncertainties encountered in automotive applications.

\section{A. Robustness of the controller during normal driving}

The first test (Fig. 4, Fig. 5 and Fig. 6) was carried out with the goal of verifying the robustness of the controller during normal driving. The lateral acceleration is less than $4 \mathrm{~m} / \mathrm{s}^{2}$. Longitudinal velocity is almost constant $(13.5 \mathrm{~m} / \mathrm{s})$ with a variable curvature between $-0.02 \mathrm{~m}^{-1}$ and $0.09 \mathrm{~m}^{-1}$. Fig. 4 shows the longitudinal speed variations. Fig. 5 presents

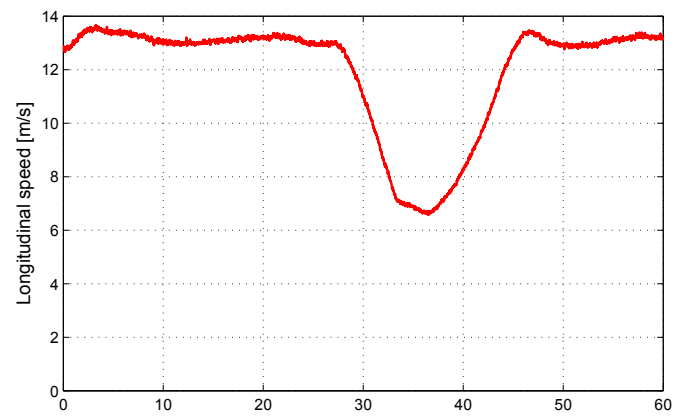

Fig. 4. Longitudinal speed

different curves. The reference path and the trajectory followed by the controlled vehicle. We have also the lateral deviation and the yaw error. The controlled vehicle is able to track the reference path with small error under various conditions. The displacement from the guideline not exceed $7.5 \mathrm{~cm}$ in transient state in this test conditions.

Fig. 6 presents dynamic variables of vehicle: the steering angle, the yaw rate and the lateral acceleration. We compare real data with data given by the simulated closed-loop system. Dynamic variables are very close to measured ones. The steering angle is smooth and the steering error does not exceed 1.7 degrees (comparing the measurement). Measured yaw rate is very close to the one obtained in simulation. We note the appearance of a small offset after the great turning. This is due to the non-linearity caused by the large steering. In this scenario, although the assumption of small angles is not respected (the steering angle is greater than 12 degrees while turning), the controller is able to follow the path with low error. This first simulation shows the good performance and robustness of the controller.

The second test (Fig. 7 and Fig. 8) was carried out with the goal of verifying the robustness of the controller during normal driving at high and varying speed. Longitudinal speed varies between $5 \mathrm{~m} / \mathrm{s}$ and $25 \mathrm{~m} / \mathrm{s}$. Note that the maximal lateral acceleration is $5 \mathrm{~m} / \mathrm{s}^{2}$. Although in this scenario we have some manoeuvres at low speed (large cornering) and at high speed, the lateral displacement is smaller than $8.5 \mathrm{~cm}$. These two first simulations show the good performance of the controller during normal driving at high and varying speed.
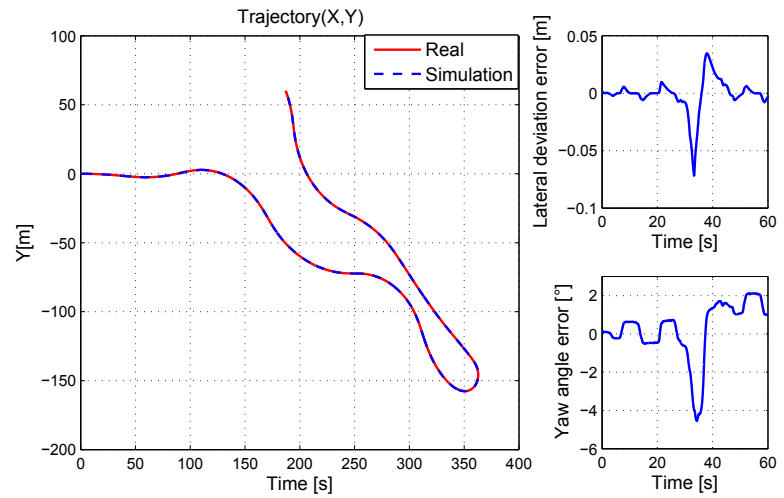

Fig. 5. Trajectories: Real (reference) and simulation (control law)
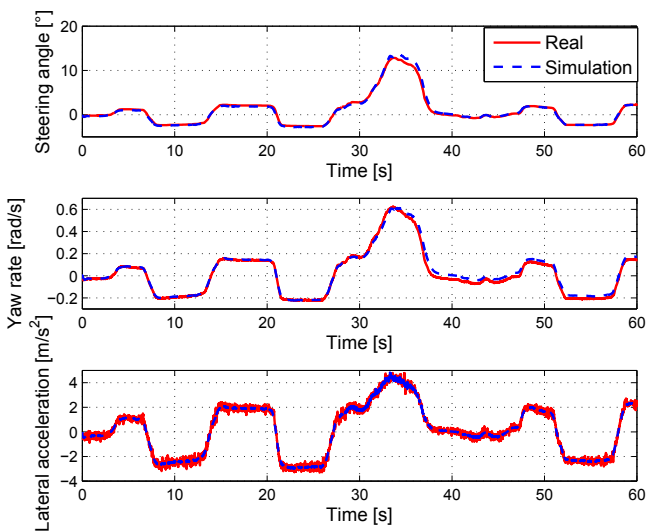

Fig. 6. Lateral acceleration, Steering angle and yaw rate

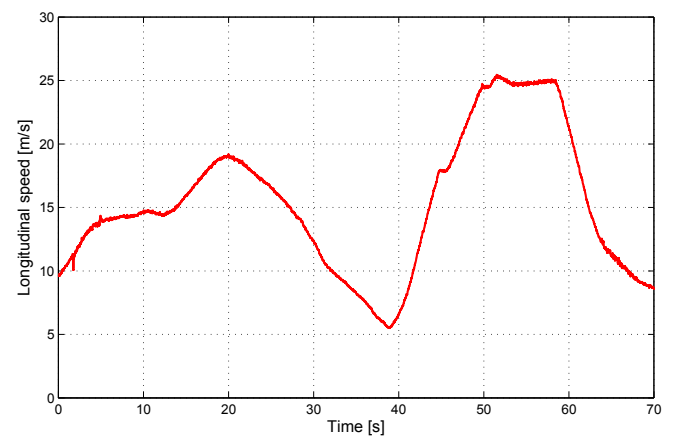

Fig. 7. Longitudinal speed
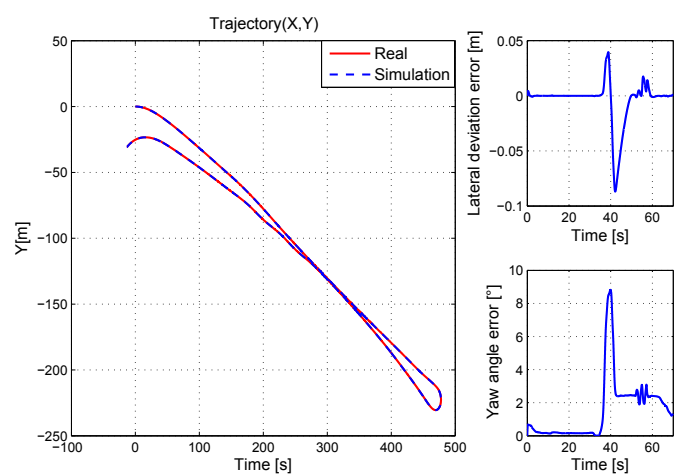

Fig. 8. Trajectories: Real (reference) and simulation (control law) 


\section{B. Robustness of the controller to strong nonlinear dynamics}

The third test (Fig. 9, Fig. 10 and Fig. 11) consists on gradually increasing speed while executing nearly the same curvature (we fixed the raduis of cuvature around $50 \mathrm{~m}$ ). In this case, the lateral acceleration was significantly increasing and the vehicle's behavior becomes highly nonlinear. This type of test is used to assess the stability and robustness of the control law to strong nonlinear dynamics. This test also evaluates the effect of changing the vehicle speed. Fig. 9 shows the longitudinal speed variations. Fig. 10 shows

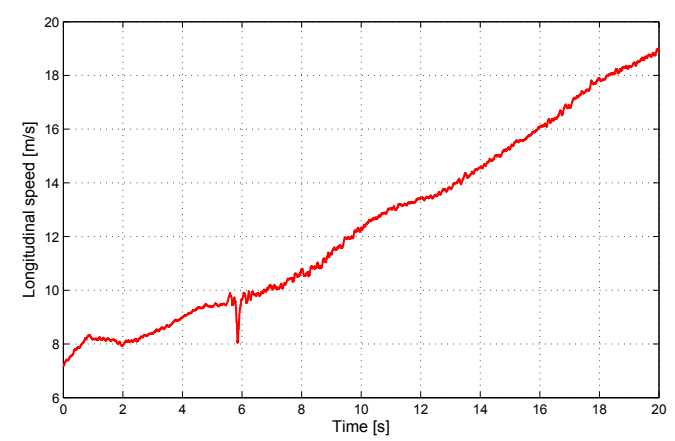

Fig. 9. Longitudinal speed

that even when the lateral acceleration is high and the large increasing of speed (rate is about $1 \mathrm{~m} / \mathrm{s}^{2}$ ), the errors remain small, when lateral acceleration reaches $6 \mathrm{~m} / \mathrm{s}^{2}$. The longitudinal speed variations have a very small influence on the maximum error.

When the lateral acceleration is higher than $7 \mathrm{~m} / \mathrm{s}^{2}$, we note a significant increase in the lateral error. In fact, the feedforward term $\left(\delta_{e q}\right)$ of the steering input is calculated using a bicycle model with linear tire forces. To improve the control performance in this case without increasing chattering, we can use a bicycle model with nonlinear tire forces to compute the feedforward term like in [24], but this complexifies the control law. Note that the maximum lateral acceleration that guarantees passengers' comfort should be limited to $4 \mathrm{~m} / \mathrm{s}^{2}$. Hence, this test aims to solicitate the vehicle beyong these limits to test the rubustness of the controller.

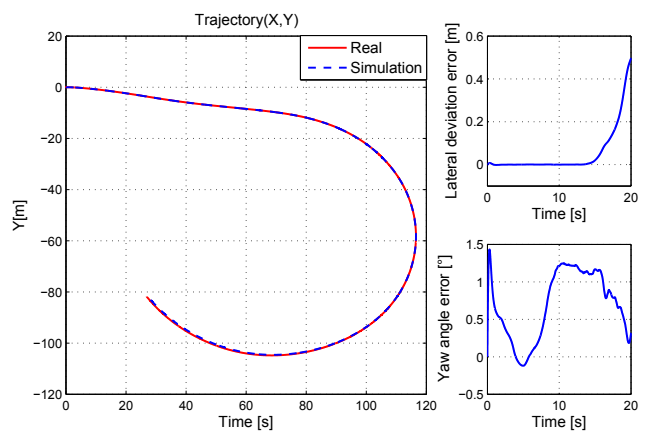

Fig. 10. Trajectories: Real (reference) and simulation (control law)

In Fig. 11 dynamic variables are very close to the measured corresponding ones even with high lateral acceleration up to $7 \mathrm{~m} / \mathrm{s}^{2}$. This test shows that the control law can ensure
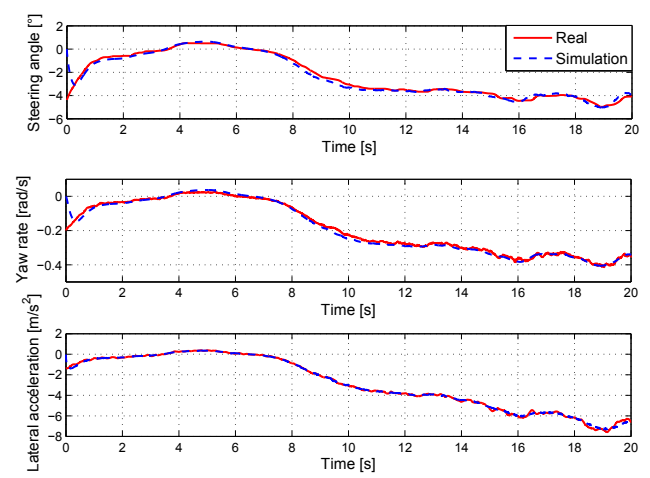

Fig. 11. Lateral acceleration, Steering angle and yaw rate

good behavior with high lateral accelarations up to $7 \mathrm{~m} / \mathrm{s}^{2}$.

\section{Robustness to vehicle parameters uncertainties}

Several parameters of the vehicle can be uncertain, for example, the cornering stiffness, the mass, etc.

It is difficult to estimate accurately the cornering stiffness of the tire. Moreover, this parameter varies greatly depending on the type of road, the vertical load, camber, etc. It is therefore important to assess the robustness of the controller over cornering stiffness variations. Fig. 12 presents lateral errors for different cornering stiffness. Despite $\mathrm{a}+/-30 \%$ of variation in the value of cornering stiffness, the controller is able to follow the path with similar errors. In other words, the controller could be able to track the trajectory (giving similar errors) with a road coefficient of friction of 0.7 ; which corresponds to a wet road. Indeed, the robustness of the controller against cornering stiffness variations, implicitly allows us to evaluate the robustness with respect to an unknown road pavement (dry or wet).

The mass of the vehicle may vary or be poorly estimated. It depends on the number of persons in the vehicle and the amount of fuel. We performed a last test concerning the robustness of the control law against the uncertainties on the vehicle mass (Fig. 13). For variations in the order of 5\%, the error remain acceptable.

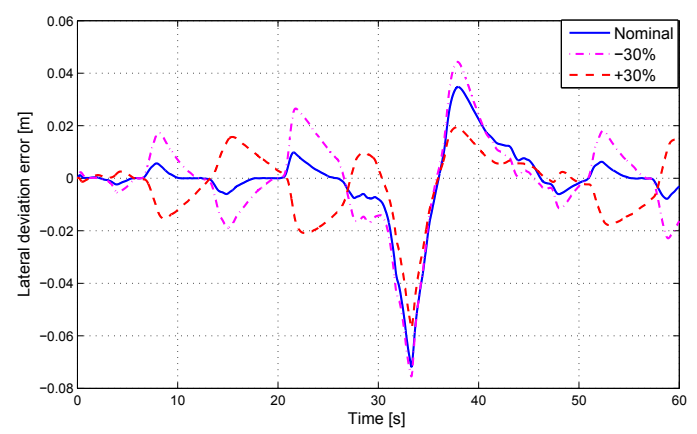

Fig. 12. Robustness against uncertainties of cornering stiffness 


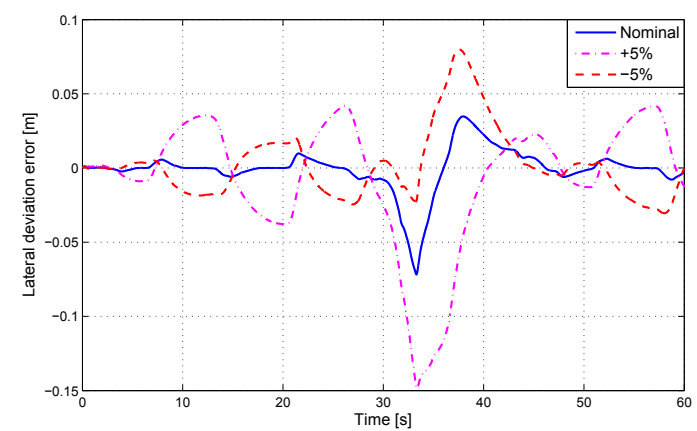

Fig. 13. Robustness against uncertainties of vehicle mass

Finally, these results show the robustness of the controller based on super twisting algorithm for autonomous driving at high speed.

\section{CONCLUSIONS}

In this paper, a strategy for lateral control of an autonomous vehicle has been developed. This strategy is based on the use of a higher order sliding mode controller -super twisting algorithm- to reduce chattering and provide robust lateral path tracking at high speed. An experimental validation has been done according to several scenarios representing different driving situations. The different tests performed highlight the robustness of the developed control law. Note that the robustness of the controlled system has been tested with respect to speed and curvature variations, and uncertain vehicle parameters.

In future work, given the implicit resemblance between the sliding mode controller and the Immersion and Invariance (I\&I) principle [25], a comparison of both controllers will be studied. The $I \& I$ theory consists on defining a target dynamics and to design a control law that renders the manifold of the target dynamics attractive and invariant. Indeed, the $I \& I$ reformulation of the stabilization problem is implicit in sliding mode control, where the target dynamics are the dynamics of the system on the sliding surface, which is made attractive by a discontinuous control law. The main distinction between both control laws is that in $I \& I$, it is not necessarily required that the manifold be reached, however, in sliding mode the manifold must be reached in finite time. This new framework $(I \& I)$ allows us to make a thorough theoretical study the robustness of the closed-loop system with respect to noisy measurements and uncertainties.

On the other hand, we will develop the control law to take into account the cant and the slope of the road. Moreover, our robotized vehicle arriving soon in the laboratory Heudiasyc, we will also test this control law on a semi-autonomous vehicle.

\section{REFERENCES}

[1] B. Siciliano, O. Khatib, and F. Groen, The DARPA Urban Challenge : Autonomous Vehicles in City Traffic, vol. 56. Springer, 2009.
[2] J. Levinson, J. Askeland, J. Becker, J. Dolson, D. Held, S. Kammel, J. Z. Kolter, D. Langer, O. Pink, V. Pratt, M. Sokolsky, G. Stanek, D. Stavens, A. Teichman, M. Werling, S. Thrun, and A. Hardware, "Towards Fully Autonomous Driving : Systems and Algorithms," in Intelligent Vehicles Symposium (IV), 2011.

[3] A. Broggi, M. Bertozzi, and A. Fascioli, "The ARGO autonomous vehicle's vision and control systems," Int. Journal of Intelligent Control and Systems, vol. 3, no. 4, pp. 409-441, 1999.

[4] P. Zhao, J. Chen, T. Mei, and H. Liang, "Dynamic motion planning for autonomous vehicle in unknown environments," in Int. IEEE Conference on Intelligent Vehicles Symposium (IV), June 2011.

[5] R. Marino, S. Scalzi, and M. Netto, "Nested PID steering control for lane keeping in autonomous vehicles," Control Engineering Practice, vol. 19, pp. 1459-1467, Dec. 2011.

[6] R. Rajamani, Vehicle dynamics and control. Springer, 2006.

[7] S. Hima, B. Lusseti, B. Vanholme, S. Glaser, and S. Mammar, "Trajectory Tracking for Highly Automated Passenger Vehicles," in IFAC World Congress, pp. 12958-12963, Aug. 2011.

[8] A. Benine-Neto, S. Scalzi, S. Mammar, and M. Netto, "Dynamic controller for lane keeping and obstacle avoidance assistance system," in Int. IEEE Conference on Intelligent Transportation Systems, pp. 13631368, Sept. 2010.

[9] M. Netto, S. Chaib, and S. Mammar, "Lateral adaptive control for vehicle lane keeping," in American Control Conference, vol. 3, pp. 2693-2698, 2004.

[10] D. Kim, J. Kang, and K. Yi, "Control strategy for high-speed autonomous driving in structured road," in Int. IEEE Conference on Intelligent Transportation Systems (ITSC), Oct. 2011.

[11] J. Kang, R. Y. Hindiyeh, S.-W. Moon, J. C. Gerdes, and K. Yi, "Design and Testing of a Controller for Autonomous Vehicle Path Tracking Using GPS/INS Sensors," in Int. Federation of Automatic Control, 2008.

[12] E. Onieva, J. Naranjo, V. Milanés, J. Alonso, R. García, and J. Pérez, "Automatic lateral control for unmanned vehicles via genetic algorithms," Applied Soft Computing, vol. 11, pp. 1303-1309, Jan. 2011.

[13] S. Chaib, M. Netto, and S. Mammar, "H inf, adaptive, PID and fuzzy control: a comparison of controllers for vehicle lane keeping," in Int. IEEE Intelligent Vehicles Symposium (IV), no. 1, 2004.

[14] T. Keviczky, P. Falcone, F. Borrelli, J. Asgari, and D. Hrovat, "Predictive control approach to autonomous vehicle steering," in American Control Conference, 2006.

[15] J. Ackermann, J. Guldner, W. Sienel, R. Steinhauser, and V. Utkin, "Linear and nonlinear controller design for robust automatic steering," IEEE Transactions on Control Systems Technology, vol. 3, pp. 132143, Mar. 1995.

[16] P. Hingwe and M. Tomizuka, "Experimental evaluation of a chatter free sliding mode control for lateral control in AHS," in American Control Conference, vol. 5, 1997.

[17] J. He, D. A. Crolla, M. C. Levesley, and W. J. Manning, "Coordination of active steering, driveline, and braking for integrated vehicle dynamics control," Journal of Automobile Engineering, vol. 220, pp. 14011420, Jan. 2006.

[18] A. Ferrara and C. Vecchio, "Second order sliding mode control of vehicles with distributed collision avoidance capabilities," Mechatronics, vol. 19, no. 4, pp. 471-477, 2009.

[19] H. Dugoff, P. Fancher, and L. Segel, "An Analysis of Tire Traction Properties and Their Influence on Vehicle Dynamic Performance," $S A E, 1970$

[20] I. Boiko, L. Fridman, A. Pisano, and E. Usai, "Analysis of Chattering in Systems With Second-Order Sliding Modes," IEEE Transactions on Automatic Control, vol. 52, pp. 2085-2102, Nov. 2007.

[21] A. Levant, "Sliding order and sliding accuracy in sliding mode control," Int. Journal of Control, vol. 58, pp. 1247-1263, Dec. 1993.

[22] A. Levant, "Robust exact differentiation via sliding mode technique," Automatica, 1998.

[23] A. Levant, "Chattering analysis," IEEE Transactions on Automatic Control, vol. 55, no. 6, pp. 1380-1389, 2010.

[24] J. Funke, P. Theodosis, R. Hindiyeh, G. Stanek, K. Kritatakirana, C. Gerdes, D. Langer, M. Hernandez, B. Muller-Bessler, and B. Huhnke, "Up to the limits: Autonomous Audi TTS," in Intelligent Vehicles Symposium (IV), June 2012.

[25] A. Astolfi, D. Karagiannis, and R. Ortega, Nonlinear and Adaptive Control with Applications. Springer, 2008 